\title{
Simulating the growth of hepatic metastases from carcinoma of the pancreas using LabVIEW
}

\author{
Alan Kawarai Lefor* \\ Department of Surgery and the Jichi Medical Simulation Center, Jichi Medical University, Tochigi, Japan
}

\begin{abstract}
The mathematical modeling of tumors has potential to contribute to individualized therapy for a wide range of malignancies. There have been many approaches to this complex subject over the last 50 years or more. The current study is an attempt to adapt a model, originally developed for neuro-oncology, to the understanding of hepatic metastases from pancreatic carcinoma. The Diffusion-Proliferation (DP) model uses the sum of a diffusion term and a proliferation term to calculate changes in tumor cell concentration over time. The model was adapted directly from previous investigators who have used it to study brain tumors. The model is based on two parameters, a diffusion parameter $\left(\mathrm{D}_{\mathrm{i}}\right)$ and a proliferation index $(\mathrm{p})$. LabVIEW is a widely used software package, which enables simulation of physical systems, especially in engineering applications, and has been applied to the diagnosis and treatment of malignancies. This is the first study to use LabVIEW for the simulation of tumor growth. The simulation allows direct entry of the two parameters, $\mathrm{D}$ and $\mathrm{p}$, with the resulting tumor growth curve appearing as a graph. The user has a rapid graphical understanding of the impact of the two parameters, $\mathrm{D}$ and $\mathrm{p}$, on tumor growth. The next step in the development of this model will be to use patient-specific data to understand tumor growth and calculate survival based on the parameters $\mathrm{D}$ and $\mathrm{p}$.
\end{abstract}

\section{Introduction}

The incidence of adenocarcinoma of the pancreas is stable in many countries although it is increasing in some developed countries where it was formerly low. This dreaded disease remains one of the most difficult malignancies to treat and cure. It is often diagnosed in late stage for a wide variety of reasons, and overall about $95 \%$ of patients die of their disease within five years. The global incidence is about $8 / 100,000$ people and the incidence in the USA is about $13 / 100,000$. Incidence rates vary about five to seven-fold from countries with the lowest to highest incidences [1].

One of the latest approaches to solving problems in biomedicine is to apply the methods of other established investigative fields. This concept has recently led to the development of the entire field of Computational Oncology [2]. The idea is to leverage the physical sciences and mathematics to solve problems in oncology, by using a sound mathematical foundation for approaches to oncologic problems. This has led to several approaches to tumor modeling, including mechanistic models, cellular models and descriptive models.

\section{Mechanistic tumor models}

Mechanistic models have been developed for pancreatic cancer [3]. Investigators have developed a complex model based on signaling pathways in cancer. Specifically, they deal with the pathways for apoptosis, cell cycle arrest and proliferation. This impressive model includes several major signaling pathways, including the Hedgehog, WNT, KRAS, RB-E2F, NFkB, p53, TGF- 3 , and apoptosis pathways. The signaling pathways are translated to a Boolean network model, designed with many nodes. The nature of the interactions among the nodes is based on studies of cell function from the laboratory. Each node is a protein or lipid in the signaling pathway. Symbolic Model Checking is then used to verify that the pancreatic cancer cell model satisfies temporal logic properties. The effect of each of the pathways coded can then be evaluated, leading cells to apoptosis, proliferation or cell cycle arrest. This is a very sophisticated model of a single cell, based on known pathways that allows investigators to study the effects of specific signaling pathways on cell activity. It is hoped that this cellular-level insight will be translated into clinically useful therapeutic approaches.

\section{Cellular growth models}

While mechanistic models as described above, are attempting to "construct" in silico tumors by modeling individual specific cellular processes, the descriptive models described below are simulating the clinically observed growth of tumors and using that to predict clinical outcomes. Between these two approaches, lie a group of models which we refer to as "cellular models" where the growth of groups of cells is described mathematically [4-6]. The importance of angiogenesis and the blood supply to tumors is a key feature of some of these models. Shirinfard and colleagues developed a three-dimensional simulation of vascular tumor growth which builds a lattice of vessels to which tumor cells are added [4]. Cells proliferate and then respond to the resulting hypoxia as demand outstrips supply. The tumor cells go through three phases of growth patterns. This innovative model allows investigators to study how tumor induced angiogenesis affects the growth of tumors. Another cellular model focuses on heterogeneous cellular interactions on tumor progression using a web-based visualization tool [5]. This system uses the Cellular Potts Model, which is based on individual cells modeling on the tissue level. More recently, investigators are using non-linear modeling to study the effects of morphology instabilities on

Correspondence to: Alan Kawarai Lefor, Department of Surgery and the Jichi Medical Simulation Center, Jichi Medical University, Tochigi, Japan; E-mail: alefor@jichi.ac.jp

Received: July 28, 2017; Accepted: August 31, 2017; Published: September 04, 2017 
both avascular and vascular solid tumor growth [6]. These investigators use a boundary-integral approach to demonstrate that morphologic instability is a means for tumor invasion. All of these approaches have the potential to further our understanding of factors affecting tumor growth.

\section{Descriptive tumor models}

The last type of tumor model used is a descriptive tumor model, which is based on patient-based clinical data rather than cellular pathways elucidated in the laboratory. Descriptive tumor models have been used with some success in the field of neuro-oncology, and led to the new discipline of Mathematical Neuro-Oncology [7]. This field uses descriptive tumor models to predict and quantify response to therapy. Advocates of this approach refer to this as precision medicine that uses patient-specific data to tailor therapy. The application of descriptive models to carcinoma of the pancreas is a relatively new approach [8].

\section{Cellular diffusion}

Tumor growth can be from cell proliferation or from diffusion of cells into the parenchyma. The first half of the model is based on cellular diffusion, using Fick's first law of diffusion. Diffusion is the net flux of particles down a concentration gradient due to random movement. For a concentration, $c$, the diffusion flux, J, is defined as:

$$
I=-D \frac{d c}{d x}
$$

where $\mathrm{x}$ is the position and $\mathrm{D}$ is the diffusion coefficient. The diffusion coefficient is the slope of the line. When $\mathrm{D}$ is large, the same gradient causes greater diffusion. In 2 or more dimensions, we use the gradient:

$$
I=-D \nabla \mathrm{c}
$$

In anisotropic media, Fick's first law uses the tensor $\mathrm{D}_{i j}$, and is expressed as the product of a vector and a tensor:

$$
I_{i}=D_{i j} \frac{\partial}{\partial x_{j}}
$$

Fick's second law (in one dimension) states that:

$$
\frac{d c}{d t}=D \frac{\partial^{2} c}{\partial x^{2}}
$$

which predicts how diffusion causes the concentration over time. We can now combine these to obtain a diffusion equation:

$$
\frac{\partial c}{\partial t}=\nabla \cdot(D(x) \nabla c)
$$

Mathematically, this is the divergence of a gradient, which known as the Laplacian. The Laplacian operator is used to generalize to more than one dimension, and when operating on a scalar field yields a scalar, the Laplacian operating on a vector yields a vector and on a tensor, yields a tensor. The Laplacian is written as:

$$
\nabla^{2}=\frac{\partial^{2}}{\partial x^{2}}+\frac{\partial^{2}}{\partial y^{2}}+\frac{\partial^{2}}{\partial z^{2}}
$$

In the diffusion model, the Laplacian is used to operate on a scalar field, and the result is a scalar, which describes the concavity of the scalar field. The diffusion component is written as:

$$
\frac{\partial c_{i}}{\partial t}=\mathrm{D}_{\mathrm{i}} \nabla^{2} \mathrm{c}_{\mathrm{i}}
$$

Here, $D_{i}$ is a constant, the diffusion coefficient. In fact, $D_{i}$ is a constant only for dilute solutions. Thus, this is one of the approximations in this model of cellular diffusion in a solid organ. Fick diffusion is based on random molecular motion, and it is difficult to mathematically estimate how much this will vary from cell diffusion in a solid organ. Nonetheless, it is a reasonable starting point.

\section{Cell proliferation}

Modeling cell proliferation is most simply carried out with an exponential model:

$$
\mathrm{y}(\mathrm{t})=N_{0} e^{\lambda \mathrm{t}}
$$

where $\mathrm{N}_{0}$ is some initial condition and $\lambda$ is a coefficient of growth. One of the problems with this simple model is that it does not reflect the fact that tumor growth is not constant, but slows down later in its growth phase.

A commonly used model for many years is the Gompertz model, which describes "Gomepertzian" tumor growth. The Gompertz model was developed in the early 1900s for insurance mortality statistics and has been applied to mobile phone uptake, population growth, finance as well as tumor growth. Application of the Gompertz model to tumor growth was first described by Laird [9] in the 1960s. The basic Gompertz model is

$$
y(t)=a e^{-b e^{-c t}}
$$

The Gompertz model accounts for the fact that tumor growth is not constant over time, but slows down toward the end of the time period $\mathrm{t}_{\max }$. A more complete expression of the Gompertz model is:

$$
\mathrm{n}(\mathrm{t})=\mathrm{N}_{0} \exp \left[\ln \left(\frac{N \infty}{N_{0}}\right)[1-\exp (-b t)]\right]
$$

The developers of the Proliferation-Invasion model, from which the DP model is directly derived, use a different approach for the proliferation term [7].

\section{The diffusion-proliferation model}

Investigators have developed a model based on the two processes of diffusion and proliferation. This mathematical model defines the rate of change of tumor cell density as equal to the diffusion of tumor cells out into the tissue plus the net proliferation of tumor cells [7]. The model was fully developed for neuro-oncology, and is referred to as the Proliferation-Invasion model. The early iteration of this model is shown here [10]:

$$
\frac{\partial \mathrm{c}}{\partial \mathrm{t}}=\nabla \cdot \mathrm{J}+\mathrm{pc}
$$

Where $c$ is the cell concentration at time $t$. The first term is the divergence of diffusion and the second term is the proliferation term. This form of the model was used in early work, but as with the exponential model described above, this model does not account for slowed tumor growth later in time. Therefore, the proliferation term was then modified to:

$$
\rho c_{i}\left(1-\frac{c_{i}}{K}\right)
$$

In this term, the use of

$$
1-\frac{c_{i}}{K}
$$

assures that as the cell concentration increases to a maximum value $(\mathrm{K})$, the growth accelerates, and then decreases, which is consistent with tumor growth. 
We will formulate the model slightly differently in the diffusion term, and shall refer to it as the Diffusion-Proliferation (DP model). In mathematical terms, it is stated as:

$$
\frac{\partial c_{i}}{\partial t}=\mathrm{D}_{\mathrm{i}} \nabla^{2} \mathrm{c}_{\mathrm{i}}+\rho c_{i}\left(1-\frac{c_{i}}{K}\right)
$$

Rate of change in Diffusion of tumor cell density tumor cells Proliferation of over time

This is a partial differential equation with two parameters: net rate of migration (D) and proliferation $(\rho)$, both of which are calculated in a patient-specific manner using clinical imaging [7]. From the point of view of modeling, the DP model is based on several approximations, especially in the diffusion term since the Fick equation is based on random molecular motion in a dilute solution. This may be a target for refinement in the future.

The success of the DP model is well documented in the modeling of gliomas. The model was used to simulate surgical resection for 70 patients with glioblastoma and predicted the survival in this group [7]. In addition to predicting tumor growth the PI model has been used to identify patients who might respond to various surgical strategies, predict response to radiation therapy and to connect clinical imaging features and genetic information [11]. While the clinical behavior of glioblastoma is not the same as adenocarcinoma of the pancreas, computational oncology may offer insights into various therapeutic approaches for patients with adenocarcinoma of the pancreas [8].

One consequence of this model is that the profile of cell concentration depends on the ratio of $\mathrm{p}$ to $\mathrm{D}$. If we vary $\mathrm{p}$ and $\mathrm{D}$, while keeping the $\mathrm{p} / \mathrm{D}$ ratio the same, then the geometry of the curves for growth and diffusion remain the same but the time scale changes [10]. This explains the need for studying tumor growth over time, since two tumors could have the same $\mathrm{p} / \mathrm{D}$ ratio at one point in time, but have very different growth characteristics depending on the particular values. For a given value of $\mathrm{D}$ and $\mathrm{p}$, using Fisher's approximation. The effects of $\mathrm{D}$ and $\mathrm{p}$ on the concentration of tumor cells over time is discussed below in relation to the LabVIEW simulation system, which graphically displays the results.

The velocity of tumor growth is easily measured from two CT scans at different times and is related to $\mathrm{D}$ and $\mathrm{p}$ by [10]:

$$
\mathrm{v}=2 \sqrt{p D}
$$

This, of course, does not allow the calculation of $\mathrm{D}$ and $\mathrm{p}$ individually, which is carried out by other means.

\section{Other models of carcinoma of the pancreas}

Haeno and Michor [12] developed a descriptive model of the growth of tumors, which begins with consideration of tumor cells in three groups, type- 0 cells which reside in the primary tumor and are not yet able to metastasize, type- 1 cells which can metastasize and type2 cells which have metastasized and form new lesions [12]. They used this model to derive several analytical approximations, including: (i) the probability of having at least one metastatic site at diagnosis and at autopsy; (ii) the expected number of metastatic sites at diagnosis and at autopsy; and (iii) the expected total number of cells in all metastatic sites at diagnosis and at autopsy [12]. By considering the cells of a tumor in these three groups and having analytic approximations to relate the size of each group, they can study the effects of various changes on the overall cell population. This model also allows the calculation of the effect of drug administration and tumor resection on the number of cells in each group, which can then be used to predict survival. This model predicts that tumor resection effectively prolongs survival when the tumor is large. The effect of the various parameters they use on metastatic rate, timing of the occurrence of metastases and growth of metastases can be demonstrated with this sophisticated model. This is the beginning of detailed descriptive mathematical modeling of tumor growth that can be correlated with a patient's clinical findings.

These same investigators then applied their descriptive tumor model to patients with adenocarcinoma of the pancreas [13]. In this study, they used data from 228 patients with adenocarcinoma of the pancreas and evaluated growth kinetics. The strength of this study in part comes from their use of extensive clinical data from 101 patients who underwent autopsy, and 127 patients treated with curative surgical resection followed by adjuvant therapy. Overall survival in the autopsy group was a median of $11.4 \mathrm{mo}$ and $21.0 \mathrm{mo}$ in the adjuvant group. The tumor kinetic data was developed using their model with data from the autopsy group and then validated using data from the adjuvant group.

The investigators review findings which support the notion that metastasis is a late event in the clonal evolution of pancreatic cancer [13]. By using data from patients who died of pancreatic cancer, with tumors measured at multiple time points, the study shows that an exponential growth model more accurately fits (median $\mathrm{R}^{2}=0.63$ ) the clinical growth pattern than a linear growth model. The model starts with a single cell that is not able to metastasize (type- 0 cell), which the multiplies exponentially and gains the ability to metastasize (type- 2 cell). They assume that a single genetic alteration is sufficient to confer metastatic ability to pancreatic cancer cells. These cells then metastasize at a certain rate at distant sites. The total number of cells is designated as $M_{1}$. The mathematical model then allows four possible options at this point including no treatment, surgical resection of the primary tumor, systemic therapy, or surgery plus systemic therapy. The authors studied tumor size and growth kinetics and found that a consideration of both parameters offers an opportunity to optimize the timing of therapy. This results of this study using a complex mathematical model supports the notion that most patients harbor metastatic disease at the time of diagnosis [13]. This may not be a surprise to clinicians who treat this disease on a regular basis, but the fact that the outcome of the model predicts what we know clinically is extremely promising for future development of more sophisticated computational models. The strength of this approach is that it is based on data derived from large series of patients. The greatest implication of this model is that it predicts optimal timing and type of intervention impacts survival [13].

\section{LabView in cancer research}

LabVIEW (2017 version, National Instruments, Austin TX) has been used in oncology, although it is much more widely known for its engineering and education applications. In one novel application, the image processing capabilities of LabVIEW were used to analyze cells from the breast, and then determine whether the cells have a benign or malignant phenotype [14]. The capabilities of LabVIEW are well matched to this task. LabVIEW has also been used to optimize treatment of cancer. In a system to follow eye-movement in the treatment of retro-orbital tumors using proton therapy, LabVIEW was used to control the administration of the treatment beam [15].

\section{LabView simulation of tumor growth using the DP model}

Having specified the DP model using the equation above, the LabVIEW simulation was then developed. The current version of 
the simulator represents a proof of concept and further development is underway to include actual patient data so that we can verify the model as a predictor survival. At present, the simulator has software to calculate the diffusion and proliferation terms which are then integrated over time. The user of the simulator can use simple dials to set the values of the Diffusion index (D) and the Proliferation Index (P). The effect on overall cell concentration, found by integrating the $\mathrm{dc} / \mathrm{dt}$ term is shown graphically. In Figure 1, the ratio of p/D is equal to 0.559, displayed on the front panel. This is a low ratio, and the configuration of the curve is very similar to that described previously (10, Figure 1). Once we increase the p/D ratio, as shown in Figure 2, to 16.9, the shape of the curve changes dramatically, as described (10, Figure 1). This shows that the LabVIEW implementation of the simulator is consistent with previous results.
The next step with the simulator will be to use patient survival data and tumor growth velocity to calibrate the values of $\mathrm{p}$ and $\mathrm{D}$, so that the model can be tested to predict survival. We know that the values of $p$ and D vary widely among patients with glioblastomas [16]. We suspect similar variation in hepatic metastases, but this will be important to evaluate in detail. This work is currently in progress.

\section{Conclusions and next steps}

The application of analytic techniques from the physical sciences and mathematics to the problems of clinical oncology has just begun. Data based on the mathematical modeling of pancreatic cancer is enticing, and may offer important insights into optimal clinical management. Success in this burgeoning field will require the close cooperation of physical scientists and seasoned clinicians in the coming years.

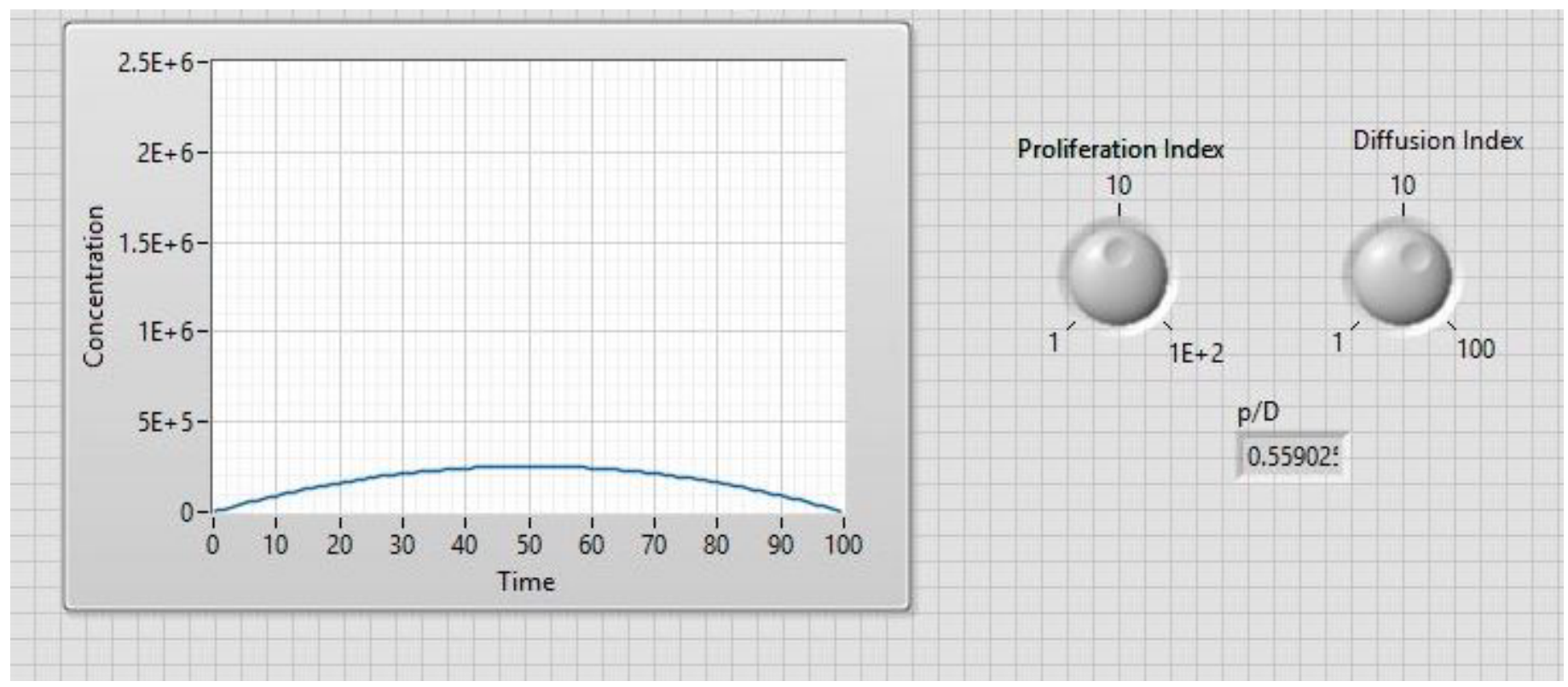

Figure 1. Cell concentration (y-axis) over time (x-axis) with a low $\mathrm{p} / \mathrm{D}$ ratio.

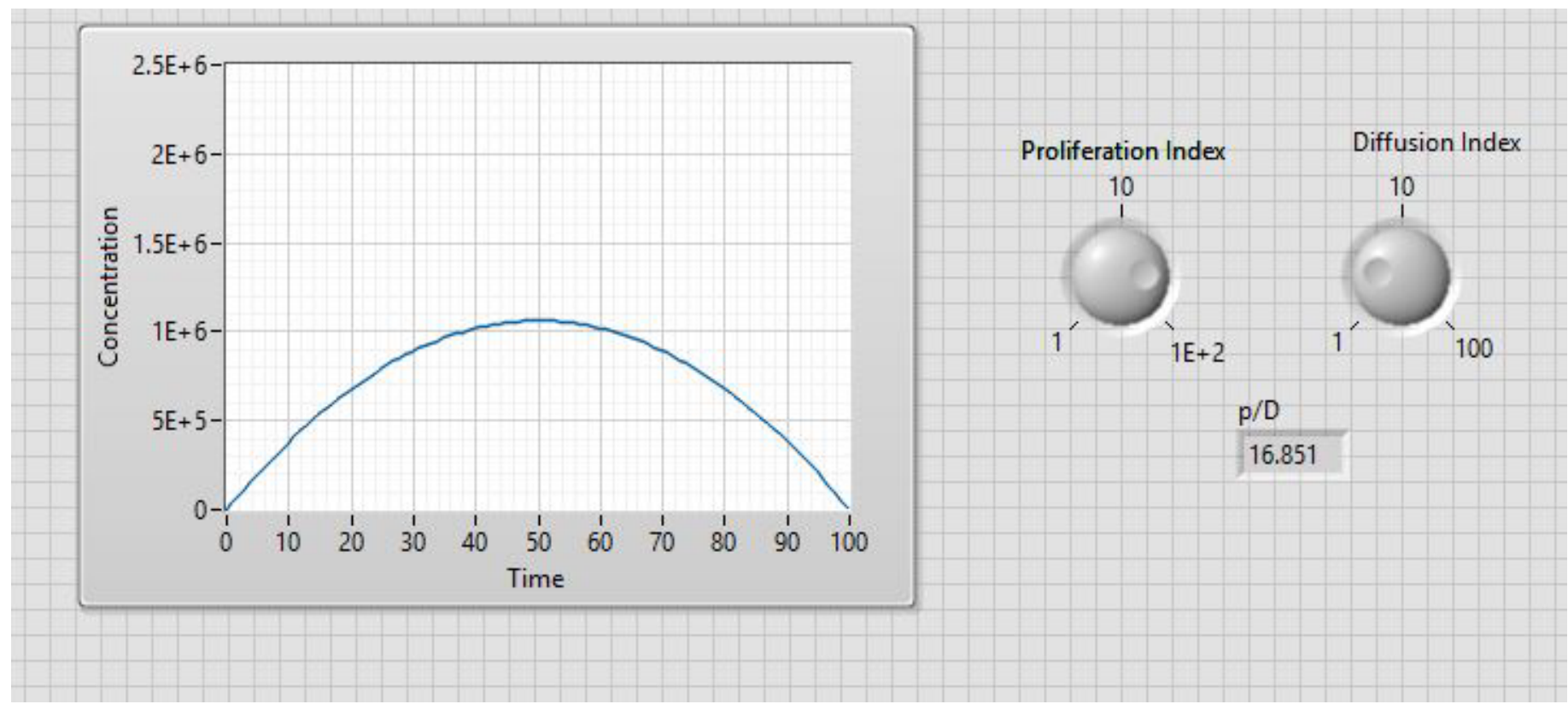

Figure 2. Cell concentration (y-axis) over time (x-axis) with a high p/D ratio. In contrast to Figure 1, as p (proliferation index) gets large relative to D, the tumor growth rate increases greatly. 


\section{References}

1. Yadav D, Lowenfels AB (2013) The epidemiology of pancreatitis and pancreatic cancer. Gastroenterology 144: 1252-1261. [Crossref]

2. Lefor AT (2011) Computational oncology. Jpn J Clin Oncol 41: 937-947. [Crossref]

3. Gong H, Zuliani P, Wang Q, Clarke E (2011) Formal analysis for logical models of pancreatic cancer. 2011 50th IEEE Conf on decision and control, Orlando FL.

4. Shirinifard A, Gens JS, Zaitlen BL, Popławski NJ, Swat M, et al. (2009) 3D multi-cell simulation of tumor growth and angiogenesis. PLoS One 4: e7190. [Crossref]

5. Jeanquartier F, Jean-Quartier C, Cemernek D, Holzinger A (2016) In silico modeling for tumor growth visualization. BMC Syst Biol 10: 59. [Crossref]

6. Cristini V, Frieboes H, Li X, Lowengrub JS, Sanga S, et al. (2008) Nonlinear modeling and simulation of tumor growth. 1-69.

7. Baldock AL, Rockne RC, Boone AD, Neal ML, Hawkins-Daaraud A, et al. (2013) From patient specific mathematical neuro-oncology to precision medicine. Front Oncol 3: 62. [Crossref]

8. Lefor AK, Maeno M (2017) Mathematical models of carcinoma of the pancreas. $J$ Pancreas 18: 216-8.

9. Laird AK (1964) Dynamics of tumor growth. Br J Cancer 18(3): 490-502. [Crossref]
10. Swanson KR, Bridge C, Murray JD, Alvord EC (2003) Virtual and real brain tumors: using mathematical modeling to quantify glioma growth and invasion. $J$ Neurol Sci 216:1-10. [Crossref]

11. Jackson PR, Juliano J, Hawkins-Daarud A, Rockne RC, Swanson KR (2015) Patientspecific mathematical neuro-oncology: using a simple proliferation and invasion tumor model to inform clinical practice. Bull Math Biol 77: 846-856. [Crossref]

12. Haeno H, Michor F (2010) The evolution of tumor metastases during clonal expansion. J Theor Biol 263: 30-44. [Crossref]

13. Haeno H, Gonen M, Davis MB, Herman JM, Iacobuzio-Donahue CA (2012) Computational modeling of pancreatic cancer reveals kinetics of metastasis suggesting optimum treatment strategies. Cell 148: 362-75. [Crossref]

14. Zadeh HG, Janianpour S, Haddadnia J (2013) Recognition and classification of the cancer cells by using image processing and LabVIEW. Int $J$ Computer Theory and Eng 5:104-110.

15. Shin D, Yoo SH, Moon SH, Yoon M, Lee SB, et al. (2012) Eye tracking and gating system for proton therapy of orbital tumors. Med Phys 39: 4265-4273. [Crossref]

16. Corwin D, Holdsworth C, Rockne RC, Trister AD, Mrugela MM, et al. (2013) Toward patient-specific biologically optimized radiation therapy plans for the treatment of glioblastoma. PLOS ONE 8(11): e79115. [Crossref]

Copyright: $\odot 2017$ Lefor AK. This is an open-access article distributed under the terms of the Creative Commons Attribution License, which permits unrestricted use, distribution, and reproduction in any medium, provided the original author and source are credited. 